\title{
Estudio exploratorio sobre eficiencia energética a empresas del Área Metropolitana de Monterrey
}

\section{Exploratory Study on Energy Efficiency for Companies in the Monterrey Metropolitan Area}

\author{
José Raúl Luyando Cuevas* \\ (D) https://orcid.org/0000-0003-0522-9368 \\ Instituto de Investigaciones Sociales \\ Universidad Autónoma de Nuevo León, México \\ jrlucu@gmail.com
}

\author{
María Florencia Zabaloy** \\ (1) https://orcid.org/0000-0003-0494-1193 \\ Departamento de Economía \\ Universidad Nacional del Sur-Instituto de Investigaciones \\ Económicas y Sociales del Sur-Conicet, Argentina \\ florencia.zabaloy@uns.edu.ar
}

* Doctor en Economía por la Universidad de Valladolid en España. Líneas de investigación: desarrollo económico y sustentable.

** Doctora en Economía. Líneas de investigación: políticas de eficiencia energética y energías renovables en Latinoamérica.

cómo citar: Luyando Cuevas, J. R., Zabaloy, M. F., Guzowski, C., Alvarado Lagunas, E. (2021). Estudio exploratorio sobre eficiencia energética a empresas del Área Metropolitana de Monterrey. Secuencia (111), e1863. DoI: https://doi.org/10.18234/secuencia.v0i111.1863 


\section{Carina Guzowski*** \\ (D) https://orcid.org/0000-0002-2745-8332 \\ Departamento de Economía \\ Universidad Nacional del Sur-Instituto de Investigaciones \\ Económicas y Sociales del Sur-Conicet, Argentina \\ cguzow@criba.edu.ar}

\section{Elías Alvarado Lagunas *** \\ (D) https://orcid.org/0000-0002-2751-7718 \\ Facultad de Contaduría Pública y Administración \\ Universidad Autónoma de Nuevo León, México}

Resumen: A nivel mundial, el sector industrial es uno de los principales consumidores de energía y, por tanto, uno de los más contaminantes. Así, aplicar medidas de eficiencia energética ( $\mathrm{EE}$ ) en dicho sector genera amplios beneficios económicos y ambientales. En este contexto, la investigación indaga el grado de implementación de medidas de EE en una muestra de empresas localizadas en el Área Metropolitana de Monterrey, en Nuevo León, México. La principal contribución del trabajo es la realización de un diagnóstico que podría ser útil para diseñar e implementar programas y acciones de EE por parte del Estado. Los resultados muestran que las grandes y medianas empresas son las que más identifican e implementan medidas de EE, dirigiendo sus esfuerzos principalmente a la capacitación de su personal. Las principales barreras que enfrentan las empresas encuestadas son de información y financiamiento, situación que se agrava en el caso de las pequeñas empresas.

Palabras clave: eficiencia energética; industria; barreras; Monterrey; México.

Abstract: The industrial sector is one of the largest energy consumers worldwide and therefore, one of the most polluting. Using energy efficiency (EE) measures in this sector therefore provides broad economic and environmental

\footnotetext{
** Doctora en Economía. Líneas de investigación: mercados de generación eléctrica en Latinoamérica, políticas de energías renovables en Argentina.

**** Doctor en Ciencias Sociales con Orientación en Desarrollo Sustentable. Líneas de investigación: desarrollo empresarial.
} 
benefits. In this context, the article examines the degree of implementation of EE measures in a sample of companies located in the Monterrey Metropolitan Area, in Nuevo Leon, Mexico. Its main contribution is a diagnosis that could be useful for designing and implementing state Ee programs and actions. The results show that large and medium-sized companies are those that most identify and implement EE measures, focusing their efforts on staff training. The main barriers faced by the companies surveyed are information and financing, particularly in the case of small companies.

Keywords: energy efficiency; industry; barriers; Monterrey; Mexico.

Recibido: 8 de junio de 2020 Aceptado: 28 de septiembre de 2020

Publicado: 3 de septiembre de 2021

\title{
INTRODUCCIÓN
}

\begin{abstract}
A nivel mundial, el consumo energético en el sector industrial representa un tercio de la demanda global de energía (IEA, 2012). Las mejoras de eficiencia energética ( $\mathrm{EE}$ ) en este sector generan beneficios no sólo para las empresas, sino también para la economía. A modo de ejemplo, a nivel empresarial se mejora la productividad, se optimizan los procesos y se generan nuevas oportunidades de negocio, mientras que a nivel nacional contribuye con la seguridad energética y la reducción de las emisiones de gases de efecto invernadero (GEI) (IEA, 2012).

Para el año 2017, el sector industrial representó $29 \%$ del consumo energético mundial, posicionándose junto con el transporte (29\%) como los sectores de mayor participación. A estos le siguen en importancia el residencial (21\%), el comercial y público (8\%) y la agricultura (2\%) (IEA, 2020a).

Dos tercios del consumo energético industrial a nivel global se explica por cuatro ramas industriales: química y petroquímica (33\%), siderúrgica (17\%), cementera (9\%) y papelera (5\%). La ventaja de aplicar políticas de EE en la industria es que, en general, el sector está conformado por un número pequeño de grandes empresas intensivas en energía, de modo que es fácil identificar a los actores clave (Fawkes, Oung, Thorpe, 2016).
\end{abstract}


En lo referente a los beneficios macroeconómicos de la EE en la industria, se pueden incluir: reducción de importaciones energéticas y, por lo tanto, una mejora de la seguridad energética, mayor desarrollo económico y creación de empleos, y la reducción de la contaminación y su consecuente contribución para mitigar el cambio climático, así como a nivel local, su efecto para mejorar la salud de la población, entre otros (Fawkes, Oung, Thorpe, 2016). Por lo tanto, existe mucha potencialidad en la EE para disminuir las emisiones de GEI en el sector industrial, dado que, como se señaló anteriormente, este es responsable de una considerable parte de las emisiones a nivel global. En efecto, según la IEA (2020b), el sector industrial representó 19\% de las emisiones de GEI a nivel mundial para el año 2017.

Durante el 2010 las medidas de eE en la industria se distribuyeron de la siguiente manera: $40 \%$ fueron programas de información, 35\% instrumentos económicos y $24 \%$ medidas regulatorias y acciones voluntarias (Tanaka, 2011). Según la IEA, las políticas regulatorias como el establecimiento de metas y los estándares mínimos de eficiencia energética cubrieron menos de $25 \%$ del uso total de energía industrial en la mayoría de las regiones en el año 2017 (IEA, 2021). En los países desarrollados se han establecido dos instrumentos para mejorar la EE en la industria intensiva en energía: el impuesto al carbono y el mercado de carbono, siendo el Sistema de Comercio de Emisiones de la Unión Europea el mejor ejemplo (Fischedick, Roy, Abdel-Aziz et al., 2014). Por otra parte, una posible medida que puede englobar un conjunto de elementos de organización en la industria es la implementación de sistemas de gestión de la energía, que son una fusión de procesos de negocios llevados a cabo por las empresas, diseñados con el objetivo de mejorar sistemáticamente la EE (Fischedick et al., 2014).

En este contexto, la presente investigación tiene el objetivo de examinar el grado de implementación de medidas de Ee en empresas (mayormente del sector manufacturero y algunas del sector servicios) ${ }^{1}$ del Área Metropolitana de Monterrey, situada al noreste de México, la cual es considerada una de las zonas de mayor industrialización en el país. Por lo que, se trata de contribuir al análisis de la EE desde una perspectiva no investigada en la región, es decir, mediante la realización de encuestas. La intención es realizar un diagnóstico,

${ }^{1}$ La EE en el sector servicios está generalmente relacionada al uso eficiente de la energía eléctrica en oficinas o locales donde prestan el servicio. Como referencia se puede consultar el estudio de SIEMENS (2010). 
con el cual se pueda constatar la proporción de las empresas encuestadas que aplica medidas de $\mathrm{EE}$, qué características poseen las empresas que implementan estas medidas, cuáles son las medidas más relevantes, etc. Aunque se debe puntualizar que el objetivo en ningún caso es medir en términos físicos la EE, es decir, la cantidad de energía efectivamente aprovechada comúnmente denominada energía útil.

Por lo que los objetivos específicos son: 1) analizar la importancia de la EE en el sector industrial, tanto a nivel mundial como nacional; 2) diseñar el instrumento a partir del cual se obtendrá la información y determinar su proceso de implementación; 3) procesar los datos obtenidos, y 4) interpretar los resultados encontrados y elaborar conclusiones.

Para tal propósito, el trabajo se estructura de la siguiente manera. En el siguiente apartado se expone una revisión breve de las principales políticas de EE en el sector industrial en México, y posteriormente se realiza una breve reseña similar, pero para el caso de Nuevo León. En el apartado subsiguiente se analizan las distintas metodologías utilizadas en la literatura para realizar estudios de EE en el sector industrial. Posteriormente, se abordarán los aspectos metodológicos de la presente investigación. Luego, se presentan los resultados generales de las encuestas realizadas, además de los datos según el tamaño de las empresas encuestas. Finalmente se aborda la discusión y las reflexiones finales.

\section{LA EFICIENCIA ENERGÉTICA Y EL SECTOR INDUSTRIAL EN MÉXICO}

En primer lugar, es importante destacar que una estrategia de EE incipiente en México tuvo sus inicios en 1989, con la llamada Comisión Nacional para el Ahorro de la Energía (CONAE), que actuaba como un órgano técnico de consulta. Diez años más tarde se reestructuró para ser un órgano administrativo desconcentrado de la Secretaría de Energía y con la entrada en vigor en 2008 de la Ley para el Aprovechamiento Sustentable de la Energía cambió su nombre a Comisión Nacional para el Uso Eficiente de la Energía (ConUeE). Cabe señalar que en la Ley para el Aprovechamiento Sustentable de la Energía se define a la EE como: 
Todas las acciones que conlleven a una reducción económicamente viable de la cantidad de energía necesaria para satisfacer las necesidades energéticas de los servicios y bienes que requiere la sociedad, asegurando un nivel de calidad igual o superior y una disminución de los impactos ambientales negativos derivados de la generación, distribución y consumo de energía. Queda incluida dentro de esta definición, la sustitución de fuentes no renovables de energía por fuentes renovables de energía" (Cámara de Diputados, 2008).

Más específicamente, para la empresa se define de la siguiente manera: "La utilización de tecnologías que requieren una menor cantidad de energía para conseguir el mismo rendimiento o realizar la misma función”, por lo que se centra en la tecnología, el equipamiento o la maquinaria utilizada en edificios y el modo de actuar de las personas para utilizar menos energía, ${ }^{2}$ aunque sin reducir el nivel de calidad o bienestar.

Algunos de los frutos cosechados por la Comisión durante estos años de operación los señala la Asociación Mexicana de Empresas de Eficiencia Energética (AMENEER, 2017):

Desde hace 30 años, en México se han desarrollado planes y programas para mejorar el consumo y ahorro de la energía en los sectores doméstico, comercial e industrial, incluyendo a las dos empresas productoras de energía del gobierno federal: Petróleos de México (Pemex) y la Comisión Federal de Electricidad ( $\mathrm{CFE}$ ). Estas medidas han contribuido a la evolución en el comportamiento de la demanda provocando ahorros sustanciales y promoviendo la $\mathrm{EE}$ del país. A pesar de esto, el potencial existente es todavía de gran tamaño, lo cual plantea buenas oportunidades.

La Comisión Nacional para el Uso Eficiente de la Energía (ConueE, 2019), señalaba: "El titular de la ConueE, Ing. Odón de Buen R., señaló que, durante los últimos 25 años, los programas, medidas y acciones de EE desarrolladas en México han contribuido más a mitigar el cambio climático que las energías renovables."

2 Support and Training for an Excellent Energy Efficiency Performance (steeep). Eficiencia energética: introducción para la empresa. Recuperado de https://www.camara.es/sites/ default/files/generico/steeep_training_material_for_smes_spanish_0.pdf 
El sector industrial representa uno de los principales motores de crecimiento de la economía mexicana, aportando más de una sexta parte del valor agregado, pero también es uno de los mayores consumidores de energía en el país (35\% del total en el año 2017) de acuerdo con la base de indicadores de eficiencia energética (BIEE) publicada por la CONUEE. ${ }^{3}$ Respecto a la evolución del consumo energético industrial, en la gráfica 1 se puede observar una tendencia al alza en el periodo 2000-2017, principalmente a partir del año 2010.

En este sentido, la Ley de Transición Energética estableció la viabilidad de Acuerdos Voluntarios entre la Secretaría de Energía y las empresas energo-intensivas del sector productivo para reducir la intensidad energética en sus actividades. De acuerdo con el informe de evaluación del programa en 2019, se establecieron dos acuerdos con las empresas Nestlé México, S. A. y Altos Hornos de México, S.A.B (Sener y Conuee, 2019a).

Asimismo, en el país existen diversos estándares mínimos de eficiencia energética a partir de la promulgación, en 1992, de la Ley Federal sobre Metrología y Normalización. Esta establece la creación de Normas Oficiales Mexicanas (NOM) sobre productos, sistemas, procesos, métodos, instalaciones y servicios. Existen diversas Nom desde los años noventa, pero han crecido notablemente en los últimos años. A modo de ejemplo, en 2017 se lanzaron aproximadamente 30 NOM (SENER y CONUEe, 2017). Si bien este tipo de medidas pueden considerarse transversales a toda la economía, impactan principalmente en el sector industrial por el tipo de equipamiento que utilizan.

Finalmente, cabe destacar el Programa Nacional para Sistemas de Gestión de Energía, que en un principio estaba destinado al sector público (administración pública nacional y empresa pública Petróleos Mexicanos), pero luego se amplió a todos los sectores de la economía -principalmente a las industrias y empresas privadas-. Estos sistemas son una metodología para lograr la mejora sostenida y continua del desempeño energético en las empresas de una forma costo-efectiva. A través de la ConUEE y el apoyo internacional se promovieron y crearon redes de aprendizaje y grupos de trabajo en diversos sectores y ámbitos, que incluyen el público y el privado, el nacional y el internacional, y el de diversos sectores usuarios de energía (SENER y ConueE, 2019b).

${ }^{3}$ Conuee. Base de Indicadores de Eficiencia Energética (BIEE): Base de Datos. Recuperado de https://www.biee-conuee.net/index.php [consulta: 10 de febrero de 2020]. 
Gráfica 1. Evolución del consumo energético industrial y de la rama manufacturera en México, 2000-2017 (petajoules)

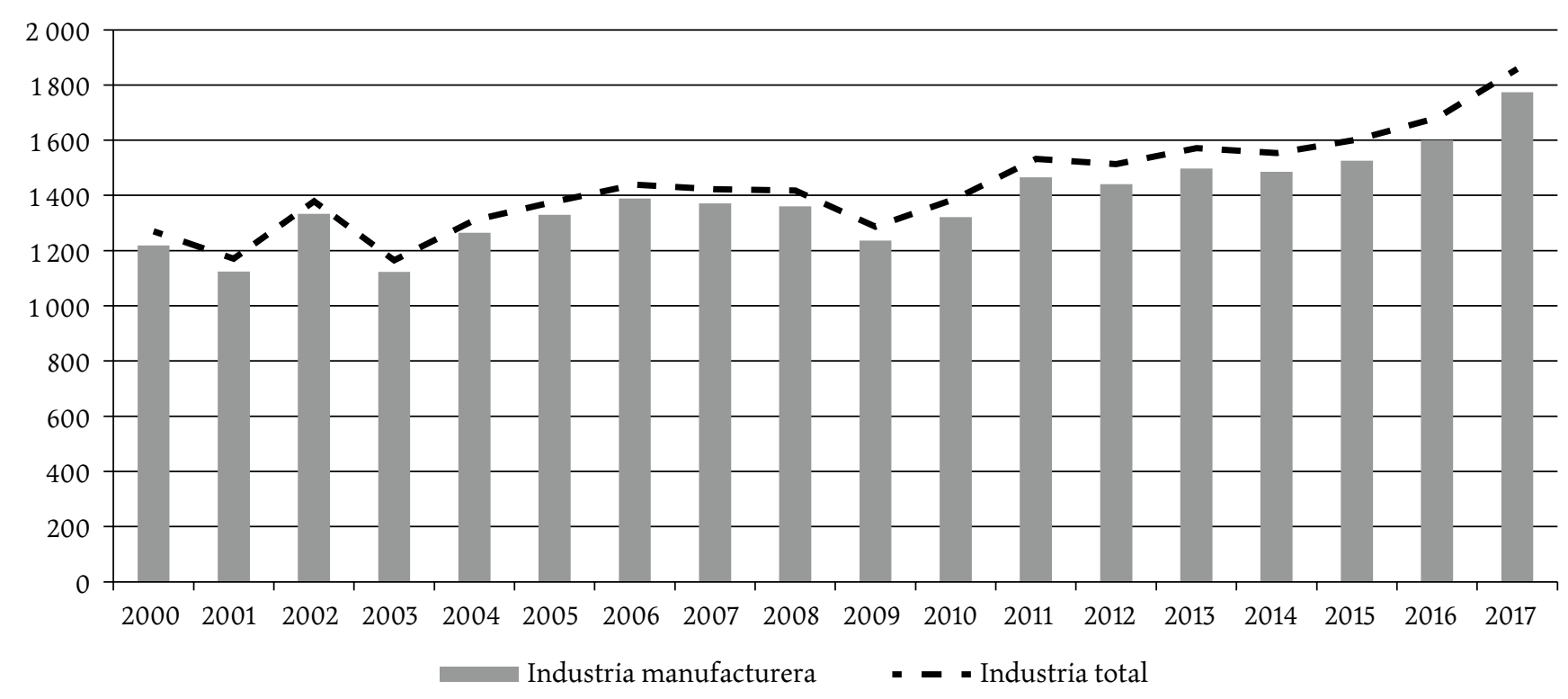

Fuente: elaboración propia con base en datos de la CONUEE. Base de Indicadores de Eficiencia Energética (BIEE): Base de Datos. Recuperado de https://www.biee-conuee.net/index.php [consulta: 10 de febrero de 2020]. 
Si bien existen diversos impulsos para la EE en la industria en México, el país sigue enfrentando desafíos. En este sentido, según Bouille, Carpio, Di Sbroiavacca et al. (2018) el sector productivo no logra asociar el vínculo entre la EE y su impacto (positivo) a nivel medioambiental; tampoco se logra visualizar los beneficios que generan las medidas de EE; y se evidencia una falta de coordinación en la promoción de medidas de EE por parte del sector público.

\section{La eficiencia energética y el sector industrial en Nuevo León}

Nuevo León es uno de los estados que más contribuye al producto interno bruto (PIB) de México, junto con la Ciudad de México, el Estado de México y Jalisco. En estos lugares se ubican las áreas metropolitanas más importantes del país, que se destacan por integrar en su estructura económica conglomerados industriales y de servicios altamente dinámicos (Ramírez y Luyando, 2014). El sector más importante del estado es el de las industrias manufactureras, las cuales concentran cerca de la cuarta parte del PIB estatal. Estas industrias aportaban $22.12 \%$ del PIB del estado en 2003 en pesos corrientes; esta contribución aumentó a 24.84\% en 2014 (INEGI, 2016).

Respecto a la EE, Nuevo León se destaca por desempeñar un papel relevante en lo relativo a este tipo de programas a nivel nacional. Entre los años 2013 y 2014 en México se aprobó una ley de reforma energética que tuvo como objetivo aumentar la productividad, la competencia y la eficiencia, en particular en los subsectores de generación eléctrica y de hidrocarburos. La Secretaría de Energía (SENER), en su Estrategia Nacional de Energía (ENE) 2014-2028, presentó un nuevo modelo energético que incluyó a la EE como un área prioritaria de transformación para ayudar a reducir la demanda de energía y la vulnerabilidad del país. Además de que con ello se contribuía a reducir las emisiones de GEI en todos los sectores y niveles de gobierno, incluyendo a los gobiernos locales. En este sentido, la SENER, con el apoyo del Banco Mundial, desarrolló en Monterrey un diagnóstico de EE con el fin de analizar el desempeño de los distintos sectores municipales en el consumo de energía, a efecto de priorizar áreas de investigación e impulsar un conjunto de medidas de EE que le permitiría al municipio desarrollar un programa al respecto. En el caso de Monterrey, los tres sectores prioritarios para la implementación de este tipo de medidas fueron: alumbrado público, residuos sólidos y edificios municipales (SEner y Banco Mundial, 2019). 
Por otro lado, el Programa Nacional para Sistemas de Gestión de la Energía, mencionado anteriormente, tuvo un importante impacto en Monterrey. En efecto, entre marzo de 2018 y marzo de 2019 se constituyó una red de aprendizaje del clúster automotriz de Nueva León, en la cual participaron ocho empresas. ${ }^{4}$ A través de distintos talleres se obtuvo una mejora en el desempeño energético, reflejado en ahorros económicos de las instalaciones participantes y se fundaron las bases para la implementación de Sistemas de Gestión de la Energía (SEner y Conuee, 2019b).

Por lo anteriormente señalado, realizar un estudio exploratorio sobre la EE en las empresas del Área Metropolitana de Monterrey resulta relevante, para entender, en líneas generales, cómo, cuándo, quién, dónde y bajo qué circunstancias se han implementado acciones de EE en dichas empresas.

\section{ANTECEDENTES EN LA MEDICIÓN DE LA EFICIENCIA ENERGÉTICA: ESTUDIOS CUANTITATIVOS VS. EXPLORATORIOS}

A nivel internacional existe diversa literatura en torno a la medición de la EE en el sector industrial con enfoques muy variados. Sin embargo, a grandes rasgos se podría afirmar que existen dos tipos de análisis: los estudios cuantitativos, que estiman el grado de EE alcanzado, y los estudios cualitativos, donde se estima de manera exploratoria cuáles son las principales medidas de EE implementadas en el sector y cuáles son las barreras específicas que explican el bajo nivel de implementación de la EE. Estos últimos, se basan en casos de estudio a nivel local y suelen utilizar encuestas o entrevistas como instrumentos para obtener la información.

En el cuadro 1 se presenta una revisión bibliográfica de los principales antecedentes en estos dos enfoques. Como se puede observar, dentro de los estudios cuantitativos predominan las herramientas: metodologías de análisis de envolvente de datos y análisis de descomposición. En el primer caso se trata de considerar múltiples entradas (inputs) y salidas (outputs) y estimar la eficiencia relativa de entidades comparables en relación con la frontera de mejores prácticas (Makridou, Andriosopoulos, Doumpos, Zopounidis,

${ }^{4}$ ProlecGE, Caterpillar, Isamex, Forja de Monterrey, Qualtia Alimentos, Viakon Conductores Monterrey, Magnekon y Blackhawk. 


\section{Cuadro 1. Antecedentes en el análisis de la EE en el sector industrial}

\begin{tabular}{|c|c|c|c|c|}
\hline Categoría & Metodología & Tipo de industria & Región & Referencia \\
\hline \multirow[t]{11}{*}{ CUANTITATIVOS } & Análisis de Envolvente de Datos & Aceite vegetal & Irán & Nouri et al. (2013) \\
\hline & Análisis de Envolvente de Datos e Índice Malmquist & Varias & Europa & Makridou et al. (2016) \\
\hline & Índices de Eficiencia Energética y Análisis de Descomposición & Industria general & Suiza & Zuberi et al. (2020) \\
\hline & Índice Malmquist & Industria general & China & Qi et al. (2020) \\
\hline & Análisis de Envolvente de Datos e Índice Malmquist & Construcción & China & Xue et al. (2015) \\
\hline & Análisis de Envolvente de Datos & Industria general & China & Zhou et al. (2019) \\
\hline & Análisis de Descomposición & Siderúrgica & México & Sheinbaum et al. (2010) \\
\hline & Análisis de Descomposición & Industria general & México & González y Martínez (2012) \\
\hline & Análisis de Descomposición & Metalúrgica & México & Ozawa et al. (2002) \\
\hline & Análisis de Descomposición & Manufacturera & México & Sheinbaum-Pardo et al. (2012) \\
\hline & Análisis Factorial & Industria general & Brasil & Sola y Mota (2020) \\
\hline CUALITATIVOS O & Visitas-Auditorías & Maquiladoras & México & Munguia et al. (2018) \\
\hline \multirow[t]{6}{*}{ EXPLORATORIOS } & Cuestionario & Fundición & Suecia & Rohdin et al. (2007) \\
\hline & Encuesta & Industria general & Ucrania & Timilsina et al. (2016) \\
\hline & Encuesta de percepción & Cementera & Colombia & Herrera et al. (2017) \\
\hline & Encuesta-Modelo de condición-recurso-gestión & Industria general & China & Wang et al. (2018) \\
\hline & Encuesta & Industria general & Cuba & Nordelo et al. (2014) \\
\hline & Entrevistas semiestructuradas y cuestionarios & Fundición & Europa & Thollander et al. (2013) \\
\hline
\end{tabular}

Fuente: elaboración propia. 
2016). Mientras que en el segundo caso se trata de una herramienta para determinar los factores que influyen en el consumo de energía y sus efectos secundarios ambientales. El método de descomposición consiste en separar o descomponer energía y emisiones; o intensidades, en variables explicativas de datos agregados (Sheinbaum-Pardo, Mora-Pérez, Robles-Morales, 2012). Por ejemplo, estos autores utilizan factores de descomposición o bien variables explicativas del consumo energético final: la actividad (producto manufacturero), la estructura (la proporción del productor generado por el subsector manufacturero) y la intensidad energética (ratio consumo final de energía-producto manufacturero). Asimismo, existen trabajos que utilizan índices para medir la EE o análisis factorial.

Por otro lado, los estudios cualitativos o exploratorios suelen hacer uso de encuestas, entrevistas o visitas para obtener información, principalmente la vinculada a la organización y gestión en la industria, donde se revela información sobre las principales barreras que se enfrentan para aplicar medidas de EE y los principales impulsores o drivers. A modo de ejemplo, Munguia, Vargas-Betancourt, Esquer et al. (2018) analizan cómo es la EE en dos maquiladoras mexicanas dedicadas a la fabricación de productos electrónicos, mediante la realización de visitas/auditorías y la recopilación de datos. Los autores encuentran que la maquiladora más grande posee políticas más fuertes de EE. Rohdin, Thollander, Solding (2007) investigan las barreras e impulsores de la EE en la industria de fundición en Suecia a través de un cuestionario, encontrando que el acceso limitado al capital constituye la mayor barrera para la EE y que los principales impulsores eran contar con personas con ambición y estrategias energéticas a largo plazo. Thollander, Backlund, Trianni, Cagno (2013) estudian impulsores o drivers de mejoras en la EE y prácticas de gestión energética en la industria de la fundición en europea a partir de entrevistas semiestructuradas y cuestionarios. Los autores encuentran que las fuerzas impulsoras más relevantes se relacionaban con las dimensiones financiera y organizacional, ya que, entre otros aspectos, gran parte de las industrias carecían de una estrategia energética a largo plazo.

Por último, tanto en los estudios cuantitativos como en los exploratorios se puede observar una diversidad de industrias y de regiones del mundo que son analizadas. En el caso de México, existen antecedentes de los dos tipos, aunque principalmente del enfoque cuantitativo. En consecuencia, en el presente artículo se realizará un estudio exploratorio, con base en información primaria proveniente de la realización de encuestas, con el fin de detec- 
tar qué tipos de medidas de EE aplican una muestra de industrias de Nuevo León y qué características socioeconómicas y técnicas poseen. A su vez, se pretende detectar las principales barreras que impiden aumentar la EE en este sector económico. De esta forma, la presente investigación contribuye con la literatura, dado que no se ha estudiado esta temática en la región de Nuevo León, a pesar de ser un nodo energético e industrial clave en el país, como se ha mencionado anteriormente. Por lo cual, el presente trabajo constituye una contribución a la literatura, dado que no existen muchos trabajos respecto a este tipo de análisis cualitativo en temas de EE industrial en México.

\section{METODOLOGÍA}

Por tratarse de un estudio exploratorio, el diseño de la encuesta consistió en una serie de preguntas dirigidas a una muestra de empresas ubicadas en el Área Metropolitana de Monterrey, sobre cuestiones relacionadas con la implementación de medidas de EE en cada una de ellas, además de algunas características socioeconómicas y energéticas -en el caso de las empresas que no llevan a cabo medidas de EE o son escasas, se trata de evaluar cuáles son algunos de los factores o barreras que imposibilitan su ejecución-. Las entrevistas para realizar las encuestas se llevaron a cabo entre los meses de octubre, noviembre y los primeros días de diciembre de 2019.

Para realizar lo anterior se utilizó el Reporte Energético Preliminar, realizado por la Subsecretaría de Energías Renovables y Eficiencias Energética (2019) de Argentina, dependiente de la Secretaría de Energía. Este estudio se basó en una encuesta -que no es de dominio público-desarrollada por el Proyecto de Eficiencia Energética en Argentina, ${ }^{5}$ el cual fue financiado por el Partnership Instrument de la Unión Europea (cuyo beneficiario final fue la Secretaría de Energía), con el propósito de conocer el estado actual del consumo de energía en los sectores, las oportunidades de eficiencia energética a nivel nacional y los principales problemas para la puesta en marcha de estas opciones de EE que enfrentan los actores. Del total de preguntas

${ }^{5}$ Secretaría de Energía. Proyecto de Eficiencia Energética en Argentina para la evaluación de las barreras para la Eficiencia Energética en el Sector Industrial Argentino. Financiado por el Partnership Instrument de la Unión Europea. Recuperado de https://www.eficiencia energetica.net.ar/ 
que se encuentran en el documento, para el cuestionario sólo se consideraron aquellas que resultaban relevantes para los fines de la presente investigación, excluyendo las que fueran específicas del proyecto que se buscaba llevar a cabo en Argentina. ${ }^{6}$ Por lo tanto, atendiendo a estas consideraciones, el número de preguntas en la presente investigación fue de $16 .{ }^{7}$ Las preguntas se dividieron en tres secciones. La primera tiene como objetivo identificar las características socioeconómicas de la empresa, es decir, conocer su ubicación, sector al que pertenece, número de años en el que ha estado realizado la actividad, número de empleados, etc. La segunda sección consiste en estudiar la caracterización energética (fuentes de energía utilizadas, principales usos energéticos, etc.). Finalmente, en la última sección se realizan las preguntas sobre las medidas de EE.

Respecto a la realización de las entrevistas y obtener las preguntas del cuestionario, cabe señalar que en muchos casos se contactó a los dueños o gerentes de las empresas, pero en otros el acercamiento fue con empleados que conocían la temática, pero que no ocupaban puestos de alta dirección, a los cuales se les remarcó que en caso de no saber alguna respuesta debían consultar a alguien que tuviera el conocimiento sobre el tema en cuestión.

Para definir el tamaño de muestra es necesario conocer la cantidad total de empresas instaladas en Nuevo León. Según la Secretaría de Economía y Trabajo (2019) del estado de Nuevo León, en 2019 se contaba con 148000 unidades económicas (empresas). Por lo que, con un nivel de confianza de 95\% y un margen de error de 9.7, el tamaño de muestra debía ser de 203 cuestionarios. En otras palabras, el tamaño de muestra se puede considerar probabilístico. No obstante, dado que las entrevistas se realizaron con base en la disponibilidad que tuvieran los dueños, gerentes o empleados de las diferentes empresas (que se contactaron mediante conocidos o recomendaciones de los mismos empresarios que habían aceptado contestar las preguntas), no se puede afirmar que cada elemento de la población tuvo una probabilidad conocida distinta de cero de ser incluido en la muestra. Por lo tanto, el muestreo en última instancia se puede clasificar como de conveniencia, pues se selecciona la muestra por el hecho de que es accesible para quien realiza el estudio.

${ }^{6}$ Plan Nacional de Eficiencia Energética (PlanEEAr) que actualmente se encuentra en desarrollo en Argentina. Para más información véase https://eficienciaenergetica.net.ar/plan_ nacional.php

${ }^{7}$ El cuestionario elaborado se anexa como parte del trabajo de investigación. 
Como señala Levy y Lemeshow (2008): "Las muestras no probabilísticas se utilizan con frecuencia, especialmente en estudios de mercado y encuestas de opinión. Se utilizan porque el muestreo probabilístico a menudo requiere mucho tiempo y es caro y, de hecho, puede que no sea factible en muchas situaciones" (p. 19) [traducción propia]. En este caso fue por lo señalado por estos autores, pero además por la reticencia de los dueños de empresas en México a dar información, principalmente por seguridad y desconfianza. ${ }^{8}$

Por lo tanto, del total de los 203 cuestionarios, $27 \%$ se realizaron en el municipio de Apodaca, $26.6 \%$ en el de Monterrey, $15.8 \%$ en el de San Nicolás, $12.3 \%$ en el de Guadalupe y, en menor medida (con porcentajes de un dígito), en los de Escobedo, San Pedro y Santa Catarina.

De acuerdo con la clasificación de empresas según la cantidad de trabajadores seguida por el Instituto Nacional de Estadística y Geografía (INEGI, 2009), la muestra se encuentra dividida tal y como se puede observar en el cuadro 2.

Según el INEgI (2020), en el Directorio Estadístico Nacional de Unidades Económicas (DENUE), señala que la mayoría de las empresas en el estado de Nuevo León son microempresas. Sin embargo, en la muestra no se observa esta característica. Esto constituye una fortaleza, pues dado el nivel de ingresos que manejan las medianas y grandes empresas, estas serían las más interesadas en aplicar políticas de EE, dado que el consumo energético representa una proporción mayor de sus costos de producción y por ello es un factor de competitividad a nivel internacional, además de que poseen el poder económico para invertir en las nuevas tecnologías y se pueden publicitar ante la sociedad como una empresa preocupada por el medioambiente y el futuro del planeta.

En otras palabras, intuitivamente se esperaría que la mayor transición a tecnologías, técnicas y políticas más eficaces en EE se encontrarían en primer lugar en las grandes empresas, seguidas de las medianas, las pequeñas, y en el caso de las microempresas se esperaría que haya escasa o nula transición. De hecho, la ConueE (2018) tiene en mente esta situación como proyecto: "La Secretaría de Energía, en colaboración con la Secretaría de Economía y el apoyo técnico de la ConUEe, deberá diseñar y establecer un programa para asesorar y apoyar a la micro, pequeña y mediana empresa en la implementación de medidas de eficiencia energética, informar sobre los beneficios que

\footnotetext{
8 Aun y cuando se señalaba que era un trabajo de investigación avalado por investigadores, en este caso por la situación geográfica, de la Universidad Autónoma de Nuevo León.
} 
Gráfica 2. Principales fuentes energéticas utilizadas por la empresa (en porcentajes)

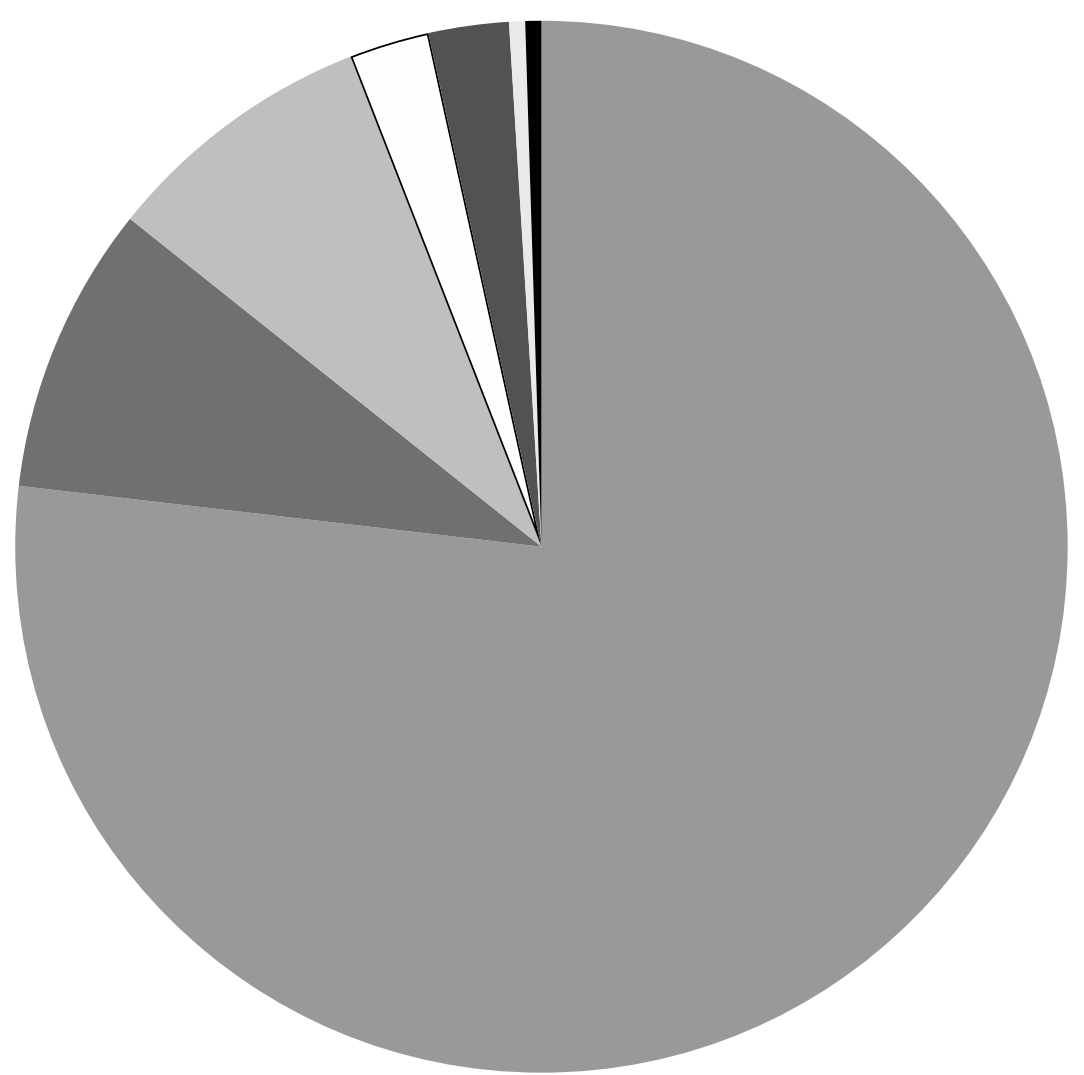

Electricidad

- Electricidad y gas natural

Gas natural

$\square$ Combustibles líquidos

\section{Otro}

Electricidad, gas natural y combustibles líquidos

- Electricidad, gas natural y carbón

Fuente: elaboración propia con base en los datos de la encuesta. 
Cuadro 2. Composición de la muestra según tipo de empresa

$\begin{array}{lr}\text { Tipo de empresa }^{\text {a }} & \text { Porcentaje } \\ \text { Micro (hasta 10) } & 2.50 \\ \text { Pequeña (entre } 11 \text { y 50) } & 28.60 \\ \text { Mediana (entre 51 y 250) } & 25.60 \\ \text { Grande (más de 250) } & 43.30\end{array}$

${ }^{a}$ Cantidad de empleados según clasificación.

Fuente: elaboración propia con base en INEGI (2009), Micro, pequeña, mediana y gran empresa.

esta conlleva, e identificar las opciones de financiamiento para que estas realicen mejoras de eficiencia energética."

\section{RESULTADOS}

Respecto a la caracterización socioeconómica de las empresas encuestadas, se detectó que la antigüedad de las empresas en la muestra es muy variada. Los años de funcionamiento van desde 1 hasta 100, con un promedio de 27 años. De forma similar, también existe una amplia variabilidad en el número de empleados, van desde 10 hasta 100 000, con una media de 1860 empleados. Un primer resultado que se puede observar respecto a estas dos variables (años de funcionamiento y cantidad de empleados) es que tienen una correlación positiva de 0.21. Por lo que se puede afirmar que hay evidencia en la muestra de la existencia de una relación lineal; en el siguiente sentido: cuanto más madura es una empresa mayor es el número de trabajadores que laboran en ella.

Respecto a la caracterización energética de las empresas, en la gráfica 2 se puede observar cuáles son las principales fuentes energéticas utilizadas por las empresas. Como se puede observar, la electricidad es una de las principales, seguida por la combinación de gas natural y electricidad y el uso exclusivo de gas natural. Estas tres alternativas juntas representan aproximadamente 94\% de las empresas encuestadas.

Con respecto a los usos o servicios energéticos de las empresas encuestadas, se puede apreciar en la gráfica 3 que los tres principales usos son: 


\section{Gráfica 3. Principales usos energéticos en la empresa (en porcentajes)}

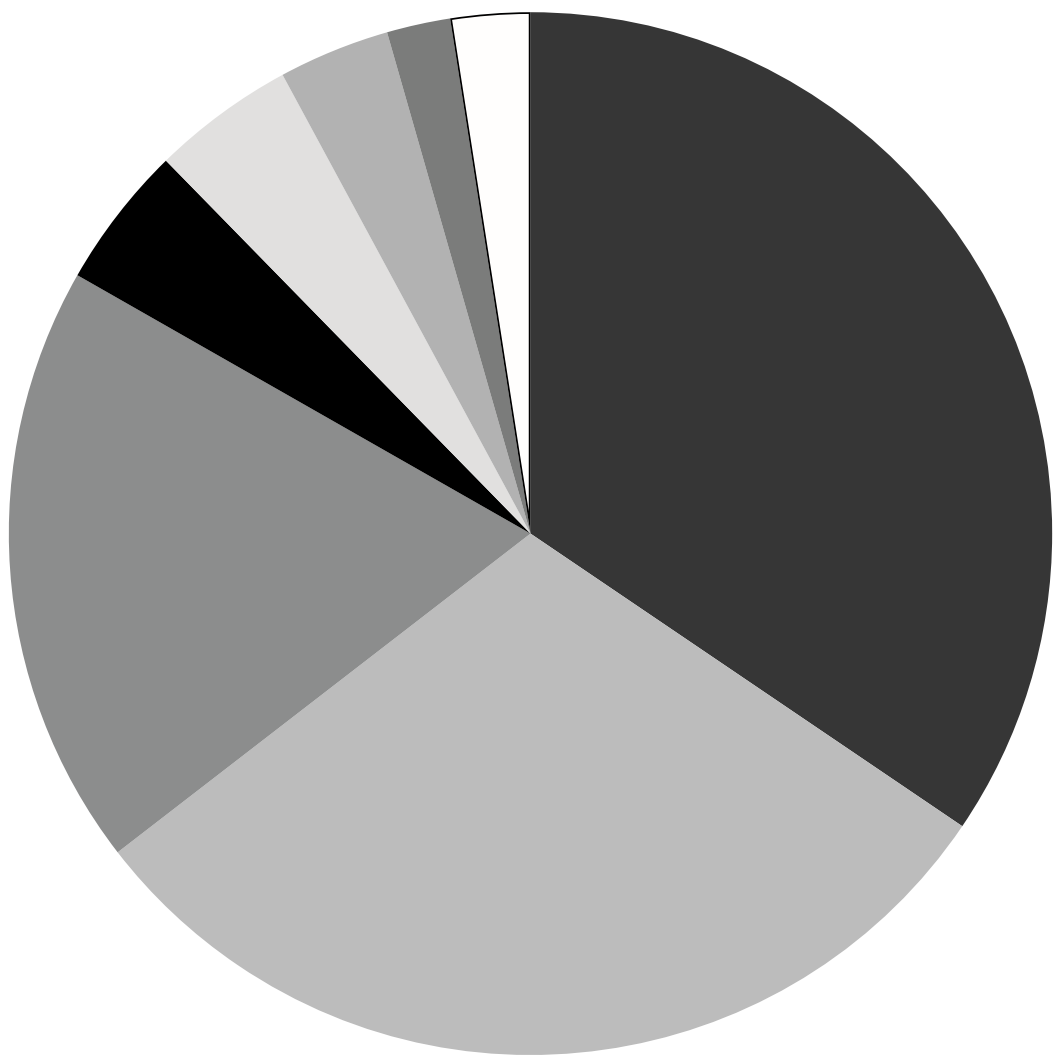

- Fuerza motriz

Fuerza motriz, iluminación y refrigeración

- Iluminación

- Calor indirecto

Calor directo

- Refrigeración

- Fuerza motriz e iluminación

$\square$ Otro

Fuente: elaboración propia con base en los datos de la encuesta. 
fuerza motriz, seguido de la opción que agrupa a fuerza motriz, iluminación y refrigeración y, por último, iluminación, los cuales representan aproximadamente $83 \%$ de las empresas encuestadas. No obstante, algunas empresas declaran utilizar calor directo e indirecto, refrigeración y la combinación de fuerza motriz e iluminación.

Otra pregunta incorporada en el cuestionario fue si las empresas conocían el impacto de los costos energéticos en sus costos de producción, a lo cual $51.72 \%$ sostuvo desconocerlo.

Sin embargo, en preguntas anteriores, $66 \%$ de las empresas habían señalado haber realizado estudios sobre el consumo de energía. En particular, estos se realizan para tener una referencia periódica del consumo energético y sus costos, por área laboral y tipo de instalación.

Además, se indagó sobre la existencia de equipos de autoproducción o cogeneración eléctrica, encontrando que $59.11 \%$ de las empresas no cuenta con este tipo de equipamiento. Ante este panorama, se consultó cuáles eran los motivos por los cuales la empresa no posee este tipo de tecnología. Como se puede observar en el cuadro 3, el principal motivo es el desconocimiento $(27 \%)$, en segundo lugar, que no hay estudios de factibilidad $(20 \%)$ y, por último, que no poseen la capacidad económica para implementarlos $(10 \%) .{ }^{9}$ En este cuadro el porcentaje más alto señaló "Otro", que junto a los que no contestaron suman $35 \%$, situación que podría estar relacionada a los que señalaron contar con este tipo de equipos.

En relación con la tercera sección del cuestionario sobre temas específicos de EE, una de las preguntas se orientó a indagar si se habían identificado potenciales acciones de EE en la empresa, a lo cual 64.53\% indicaron que sí. Las acciones específicas que identificaron se pueden observar en el cuadro 4. Las principales alternativas señaladas por las empresas fueron: mejoras en procesos, modernización de equipamiento, mejoras en la estructura y al uso eficiente de la energía, que juntas suman $59.6 \%$ de los casos. En el caso de las empresas que no identificaron ninguna acción, señalaron que no era necesario dado que la empresa aplicaba las mejores técnicas.

Además de preguntar sobre el potencial identificado de EE, se consultó si la empresa había implementado alguna acción de sobre esta. En este caso las respuestas fueron relativamente dispersas, sin embargo, presentamos las

${ }^{9}$ En los cuadros que no suman el 100\% de las respuestas, es porque sólo se incluyeron los porcentajes mayores o las respuestas más significativas. 


\section{Cuadro 3. Motivo por el cual la empresa no posee autoproducción o cogeneración eléctrica}

$\begin{array}{lccc}\text { Respuesta } & \text { Frecuencia } & \text { Porcentaje } & \text { Acumulado } \\ \text { Otro } & 65 & 32.02 & 32.02 \\ \text { Desconocimiento } & 55 & 27.09 & 59.11 \\ \text { No existen estudios sobre su viabilidad } & 42 & 20.69 & 79.80 \\ \text { No es económicamente viable } & 20 & 9.85 & 89.65\end{array}$

Fuente: elaboración propia con base en los datos de la encuesta.

\section{Cuadro 4. Potenciales acciones de EE identificadas por la empresa}

Respuesta

No es necesario, la empresa aplica las mejores técnicas o

$\begin{array}{lll}\text { procesos existentes } & 61 & 30.05 \\ \begin{array}{l}\text { Medidas asociadas a mejora de procesos (MAMP) } \\ \text { Medidas de recambio/modernización de equipamiento }\end{array} & 34 & 16.75 \\ \quad \text { (MME) } & 28 & 13.79 \\ \text { MME, Medidas asociadas a la estructura (MAE) y MAMP } & 28 & 13.79 \\ \text { Medidas asociadas al uso eficiente o racional de la energía } & 20 & 9.85 \\ \text { MAE } & 11 & 5.42 \\ \text { Total } & & 89.65\end{array}$

Fuente: elaboración propia con base en los datos de la encuesta.

principales en la gráfica 4. Como se puede observar, la mayoría de las empresas sostiene haber implementado acciones de EE relacionadas con la capacitación técnica al personal (43 empresas), seguida de mejoras en la edificación (17 empresas). En contraste, se observa que apenas ocho empresas realizan recambio tecnológico y mejora de procesos. Asimismo, se destaca que fue muy reducido el número de empresas que se han capacitado en sistemas de 
Gráfica 4. Principales acciones de EE implementadas en la empresa

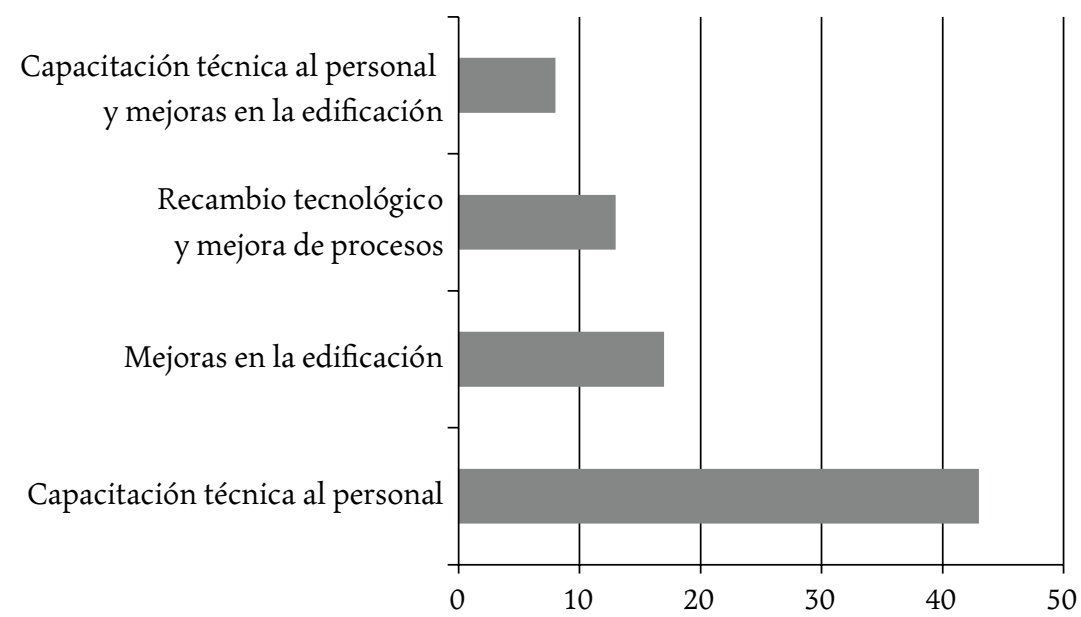

Fuente: elaboración propia con base en los datos de la encuesta.

gestión de la energía en el marco de la norma ISO 50001, razón por la cual no aparece en la gráfica mencionada.

Dentro de la sección de la encuesta referida a las medidas de EE, también se preguntó ¿cuáles son los principales problemas que enfrenta o enfrentaría la empresa para implementar acciones de EE? Las respuestas más representativas se han agrupado en la gráfica 5. En concreto, 26 empresas declararon enfrentar problemas de altos costos de las tecnologías, 25 señalaron el desconocimiento del potencial de las acciones de EE y 21 los altos costos de financiamiento. En menor medida, señalaron el problema de desconocer la participación de sus costos de energía sobre el total de costos y la opción conjunta sobre elevados costos tanto de tecnología como de financiamiento.

Por último, se indagó acerca de a quién se debían las iniciativas de EE implementadas por estas empresas. En concreto, se consultó si las mejoras de EE se debían a una iniciativa propia de la empresa o a normas, regulaciones o incentivos impulsados desde el gobierno federal. Las respuestas indicaron que $76.35 \%$ se debe a la iniciativa de la propia empresa y sólo $15.27 \%$ de los casos señalaron que se debía a una exigencia por parte del gobierno, mientras que $8.37 \%$ indicó que se debía a otras situaciones o consideraciones. 


\section{Gráfica 5. Principales problemas que enfrenta} la empresa para implementar acciones de EE

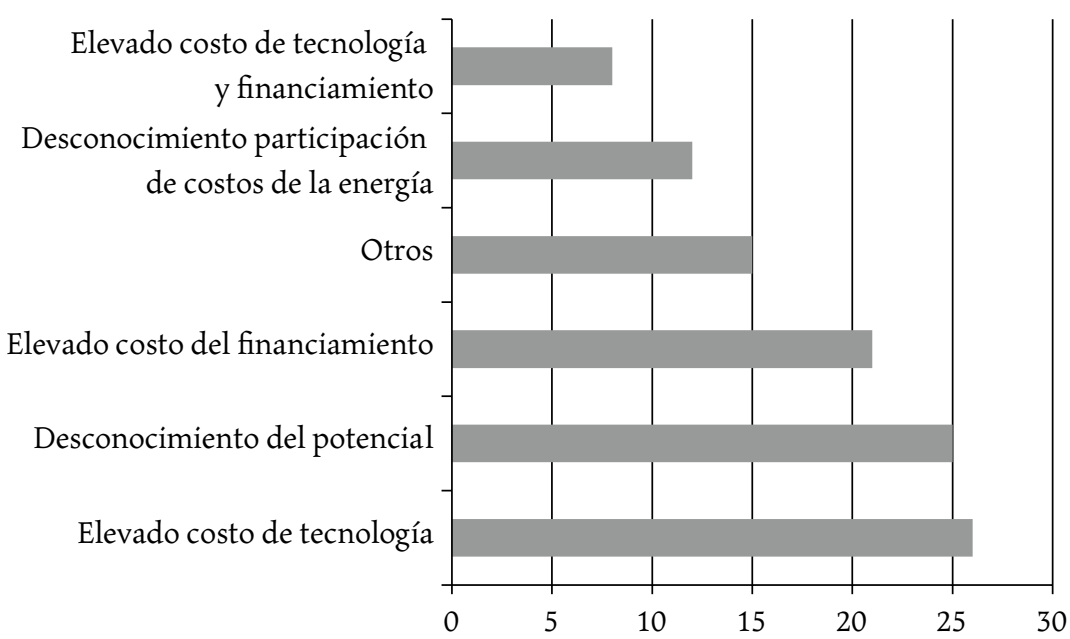

Fuente: elaboración propia con base en los datos de la encuesta.

\section{Resultados según tamaño de la empresa}

Con el fin de tener otra perspectiva de la problemática, en el cuadro 5 se presentan resultados de algunas de las principales preguntas del cuestionario de acuerdo con el tamaño de la empresa. Aunque hay pocas microempresas, es interesante observar que ninguna de ellas señaló haber realizado estudios sobre su consumo de energía. En el caso de las pequeñas lo realizaron $45 \%$, y en el caso de las medianas o grandes la mayoría señalaron haberlo realizado. A pesar de que muchas empresas declaran realizar estudios sobre el consumo de energía, no muchas conocen el impacto de los costos del consumo energético en los costos totales de producción. En efecto, en el caso de las grandes empresas, $78 \%$ señaló haber realizado estudios sobre su consumo de energía, pero sólo $42 \%$ indicó conocer el impacto de estos en sus costos de producción. En el caso de las pequeñas la relación es inversa, 52\% conocía el impacto y $45 \%$ señaló haber hecho el estudio. 
En el cuadro 5, en relación con la columna de la tercera pregunta, se puede afirmar que cuanto más grandes son las empresas, un mayor porcentaje señala tener equipos de autoproducción o cogeneración eléctrica.

En lo referente a la identificación de acciones potenciales de EE, las empresas medianas fueron las que señalaron haberlo realizado más veces (73\%)..$^{10}$ En el caso de las preguntas sobre cuáles fueron esas medidas, una respuesta frecuente fue que no eran necesarias dado que la empresa aplicaba mejores técnicas o procesos. Esto lo indicó $38 \%$ de las pequeñas empresas, $21 \%$ de las medianas y $28 \%$ de las grandes.

En la última columna del cuadro 5, respecto a la pregunta de ¿A iniciativa de quién se han aplicado mejoras de EE en la empresa?, se tiene que la respuesta mayoritaria es que la iniciativa surge de la propia empresa. A su vez, el mayor porcentaje de quienes señalan que se debe a la iniciativa del gobierno son las grandes empresas, con lo cual se puede considerar que los diferentes gobiernos han puesto mayor atención a estas por ser las que tienen más altos consumos, y además por ser de fácil acceso por su pequeño número -además de ser muy notorias en el ámbito económico nacional-. Sin embargo, se debe considerar que $7 \%$ de las medianas y grandes empresas indicaron que fueron iniciativas no relacionadas ni a la empresa ni al gobierno. ${ }^{11}$

$\mathrm{Al}$ analizar si por el tamaño de empresa se habían identificado acciones diferenciadas viables de EE, se puede señalar que los porcentajes más significativos en este sentido fueron: $13.8 \%$ de las pequeñas empresas realizó medidas de recambio o modernización de equipamiento y $12.1 \%$ implementó medidas asociadas a mejora de procesos. En el caso de las medianas, 17.3\% realizó medidas asociadas a mejora de procesos, $15.4 \%$ medidas de recambio o modernización de equipamiento y $13.5 \%$ medidas asociadas al uso eficiente o racional de la energía. En el caso de las grandes empresas, 20.5\% realizó medidas asociadas a mejora de procesos, $19.3 \%$ medidas de recambio o modernización de equipamiento, combinada con medidas asociadas a la estructura $y$ a mejora de procesos.

Ante la pregunta de ¿Cuáles son los principales problemas que enfrenta o enfrentaría para implementar acciones de EE?, las respuestas, dado

${ }^{10}$ En este caso los porcentajes que no suman 100 es porque hubo empresas que no contestaron a la pregunta.

${ }^{11}$ De nueva cuenta, en los casos en donde los porcentajes que no suman 100 , es porque hubo empresas que no contestaron a la pregunta. 


\section{Cuadro 5. Respuestas según tamaño de las empresas (porcentajes)}

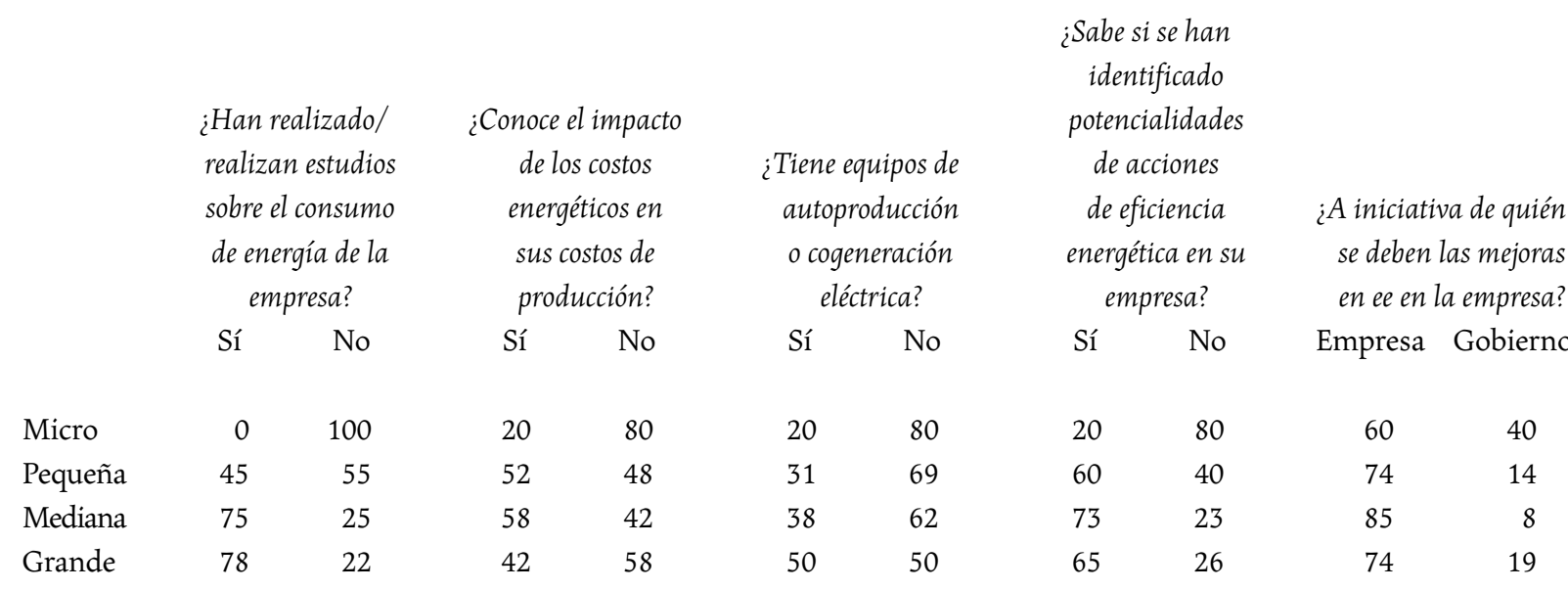

Fuente: elaboración propia con base en los datos de la encuesta. 
el tamaño de la empresa, son: las microempresas dieron respuestas muy dispersas; $12.3 \%$ de las pequeñas señaló que era el desconocimiento del potencial que tendría la EE en su empresa y esta, en combinación con otros de los problemas que enfrenta, aumenta a $28 \%{ }^{12}$ De igual manera, señalaron que otro de los problemas fue el elevado costo de las tecnologías eficientes, que alcanzó $12.8 \%$ y en combinación con otras respuestas $31 \%$. En el caso de las medianas, el desconocimiento del potencial alcanzó 7.7\% y esta, en combinación con otros problemas que enfrenta, llega a $1.2 \%$; ahora bien, en lo referente al costo es de $13.5 \%$ y en combinación $30.8 \%$. En el caso de las grandes el desconocimiento del potencial en solitario fue de $13.8 \%$ y en combinación alcanza $28.7 \%$; en lo referente al costo en solitario es $11.7 \%$ y en combinación $28.7 \%$.

El desconocimiento del potencial de la EE como barrera para su implementación parece relacionado, como se esperaría, con el porcentaje de empresas pequeñas, medianas y grandes que indicaron no saber si se habían identificado potenciales acciones de EE en la empresa. Una situación que parece se debe a falta de información y, por tanto, una que podría solventar el gobierno federal, pues estaría dentro de sus atribuciones.

En el caso de la barrera por el elevado costo de las tecnologías más eficientes, un similar porcentaje tienen las pequeñas y medianas empresas $(31 \mathrm{y}$ $30.8 \%$ ), pero las grandes no están muy distantes $28.7 \%$ (en combinación). Con este tipo de medida, se podría entrever que se trata de un problema transversal que afecta a un número similar de empresas de cada tamaño (sin considerar a las micro), pero había que investigar más minuciosamente en cada caso, para tener una perspectiva mejor, pues en solitario las respuestas de las grandes empresas sobre el desconocimiento del potencial que tendría la EE es mayor (13.8\%) al del elevado costo de las tecnologías eficientes (11.7\%), contrario a lo que pasa en el caso de las pequeñas y, sobre todo, de las medianas. Lo anterior nos podría dar algún indicio de que el problema mayor para las grandes es, en general, el desconocimiento más que el costo, situación que estaría acorde a la intuición de que las grandes deberían tener mucho menos dificultades financieras para adquirir este tipo de tecnologías (mediante sus propios ingresos o créditos en mejores condiciones, dado el volumen de dinero que manejan) y las pequeñas tendrían muchos más.

${ }^{12}$ En estas preguntas las opciones de respuesta fueron múltiples, por lo que podían escoger una o una combinación de ellas (véase formato de encuesta). 


\section{DISCUSIÓN}

En este apartado se discuten e interpretan los resultados encontrados, con el propósito de identificar las principales implicancias del estudio exploratorio realizado, tanto nivel de política pública como a nivel de contribución con la literatura científica.

Como resultados principales se encontró que $66 \%$ de las empresas encuestadas realizó estudios sobre los costos en energía, siendo muy relevantes en el caso de las medianas y grandes empresas. Sin embargo, sólo 48.28\% declaró conocer el impacto de estos costos sobre los costos totales de producción, teniendo en este caso un papel destacado las medianas empresas. A su vez, $64.53 \%$ de las empresas declaró identificar potenciales para implementar medidas de EE, especialmente en medianas y grandes empresas.

En total, aproximadamente $40 \%$ de las empresas encuestadas afirmó haber implementado alguna acción de EE. Dentro de las acciones de EE efectivamente implementadas se destaca en mayor medida la capacitación técnica de personal (21\%) y en menor medida las mejoras en la edificación (8\%).

El hecho de que haya un gran porcentaje de empresas que realizan estudios sobre los costos energéticos y que conocen el potencial de las acciones de EE, y al mismo tiempo haya un porcentaje de implementación de medidas relativamente más bajo, puede explicarse por la existencia de barreras a la EE. En la literatura se hace referencia a la denominada Brecha de Eficiencia Energética (energy efficiency gap), que es la diferencia entre el nivel actual de eficiencia energética y nivel potencial de eficiencia energética (Golubchikov y Deda, 2012; OECD, IEA, AFD, 2008). La existencia de esta brecha de eficiencia energética se debe a la presencia de las fallas de mercado o bien a las barreras de mercado para la eficiencia energética (OECD, IEA, AFD, 2008; Bouille, 1999). Las barreras específicas son mecanismos que inhiben la inversión en tecnologías que son energéticamente eficientes y económicamente rentables (Ruchansky, Januzzi, Buen, Romero, 2011).

En concreto, al analizar los problemas que enfrentan las empresas a la hora de implementar acciones de EE, se detectó que la principal falta es de información. Otra barrera detectada es el elevado costo de las tecnologías más eficientes. En relación con este aspecto, un resultado esperado hubiera sido encontrar que las grandes empresas tendrían menos problemas financiaros que las pequeñas y medianas para adquirir tecnologías eficientes, lo cual se puede entrever si sólo se considera este dato en solitario. Sin embargo, en con- 
junto con otros problemas, pareciera ser un problema transversal que afecta a varias empresas independientemente de su tamaño.

Por lo tanto, el gobierno podría reforzar las políticas públicas de información y concientización en el ámbito de la EE, así como también los instrumentos económicos que faciliten el acceso al financiamiento del equipamiento especializado. Por otro lado, a raíz del estudio realizado se pudo observar que la principal fuente energética utilizada por las empresas encuestadas es la electricidad y el principal uso energético es la fuerza motriz. En consecuencia, los esfuerzos del gobierno en los aspectos mencionados anteriormente deben dirigirse especialmente a tecnologías que utilicen electricidad y que generan fuerza motriz.

En relación con la autoproducción o cogeneración de electricidad se encontró que las grandes empresas son las que mayormente utilizan estos equipos. Pereciera que estas tendrían mayores incentivos para hacerlo, por el ahorro que implican estas fuentes de generación y quizá por la existencia de metas de reducción de emisiones de carbono. Esto último se relaciona con la imagen de la empresa como responsable con el medioambiente, ya que algunas de ellas exportan sus productos a países que tienen una alta conciencia al respecto o existen leyes en esos países que exigen este tipo de acciones. Por ejemplo, cabe señalar que "Cerca del 80\% de las exportaciones mexicanas llega a Estados Unidos, en junio del 2018 el monto generado por éstas fue de 29.9 billones de dólares". ${ }^{13}$ Pero al mismo tiempo, como lo indicó en su momento la Comisión Económica para América Latina y el Caribe (CEPAL, 1999), para el caso específico del camarón y las restricciones que impuso Estados Unidos para su importación: "La protección al medio ambiente es un objetivo legítimo de política, pero, no deja de ser inquietante, que las normas ambientales puedan convertirse en importantes barreras al comercio internacional y que se traduzcan en nuevas condiciones previas para tener acceso a los mercados de los países industrializados."

Finalmente, es importante destacar que $76.35 \%$ del total de la muestra de empresas indicó que las acciones de EE implementadas se deben a iniciativas propias, lo cual se acentúa en el caso de las empresas medianas. Por su parte, la mayor injerencia del gobierno se puede observar en las empresas

13 A. García, "Crecen las exportaciones de México hacia EU”, El Economista, 14 de agosto de 2018. Recuperado de https://www.eleconomista.com.mx/economia/Crecen-las-exportaciones-de-Mexico-hacia-EU-20180814-0031.html 
micro y grandes. Por lo tanto, también se podría recomendar que se diseñen programas de EE específicamente dirigidos a las pequeñas y medianas empresas, no sólo por los resultados vistos en relación con el tipo de iniciativas, sino también porque se ha encontrado un rol destacado de las medianas empresas en cuanto a la realización de estudios, al conocimiento de impacto de los costos energéticos y a la identificación de acciones potenciales de EE.

Por último, cabe destacar que realizar estudios exploratorios como el realizado en esta investigación representa un aporte a la literatura, ya que permite identificar barreras que contribuyen con la brecha de eficiencia energética, a partir de información cualitativa. Además, es importante remarcar la utilidad de generar este tipo de información primaria para la sociedad, ya que puede ser de gran ayuda para los hacedores de política.

\section{CONCLUSIONES}

La EE puede generar autonomía energética para la industria y la liberalización de recursos monetarios que podrían ser canalizados a la optimización de conocimientos tecnológicos al interior de los propios procesos productivos. Dada la importancia de la EE en el sector industrial, este trabajo de investigación tuvo como objetivo prioritario analizar el grado de implementación de medidas de EE en una muestra de empresas ubicadas en el Área Metropolitana de Monterrey, en el estado de Nuevo León, México.

Los resultados del trabajo reflejan que la región sigue enfrentando algunos desafíos en esta materia. De acuerdo con el estudio exploratorio realizado, se encontró que $66 \%$ de las empresas encuestadas realizó estudios de EE y cerca de $64 \%$ reconoció las potencialidades de la implementación de tales medidas. Entre las principales medidas que fueron identificadas están la mejora en procesos y el recambio o modernización del equipo, aunque la acción de EE más realizada fue la capacitación técnica al personal. Esto puede estar vinculado con la existencia de barreras a la EE.

En particular, se identificaron dos grandes barreras: la falta de conocimiento y los elevados costos tanto de la tecnología como del financiamiento. Respecto al primero, hay que recordar que anteriormente se señaló que durante el año 2010 la mayor medida de EE en la industria fueron los programas de información (40\%), de ahí la importancia de reforzar iniciativas públicas que llenen los vacíos existentes en esta materia. En el segundo caso, 
hay que profundizar más el estudio en este aspecto, pues hay indicios de que puede ser un problema de igual magnitud en los distintos tipos de empresa o uno que es más preocupante en las pequeñas y medianas empresas.

La implementación, en la mayor cantidad de empresas, de la medida de capacitación al personal en lo referente a la EE, se podría explicar por el simple hecho de que es una medida fácil y económica de aplicar.

Asimismo, se ha encontrado un rol destacado de las medianas empresas en cuanto a la realización de estudios sobre costos energéticos, al conocimiento de impacto de los costos energético en las funciones de producción y a la identificación de acciones potenciales de EE.

Por todo lo mencionado, a nivel de política pública se recomienda reforzar los instrumentos de información y concientización, por un lado, y los instrumentos económicos que faciliten el acceso al financiamiento, por otro. A su vez, podrían generarse programas específicos para las medianas empresas, ya que han demostrado tener un fuerte dinamismo en el área de la EE. Por último, estos instrumentos de política deberían focalizarse en medidas de EE que impacten en el consumo de electricidad y en el uso de la fuerza motriz, dado que son la principal fuente energética y servicio energético utilizados en el sector. En este sentido, el gobierno también podría fomentar que las industrias autogestionen su propia producción eléctrica a través de emprendimientos que involucren fuentes renovables de energía y de esta manera coordinar políticas de EE y de energías renovables en Nuevo León y especialmente en el Área Metropolitana de Monterrey.

\section{LISTA DE REFERENCIAS}

Ameneer [Asociación Mexicana de Empresas de Eficiencia Energética] (2017). ¿Por qué ahorrar energía? Mercado de eficiencia energética en México. Recuperado de http://ameneer.org.mx/porque-ahorrar-energia/

Bouille, D. (1999). Lineamientos para la regulación del uso eficiente de la energía en Argentina. Proyecto CEPAL/Comisión Europea "Promoción del uso eficiente de la energía en América Latina”. (Serie Medio Ambiente y Desarrollo 16). Recuperado de https://www.cepal.org/es/publicaciones/5701-lineamientos-laregulacion-uso-eficiente-la-energia-argentina

Bouille D., Carpio C., Di Sbroiavacca N., Dubrovsky H., Nadal G., Lallana F., Landaveri R., Pistonesi H., Plauchú J., Recalde M. y Soria R. (2018). Propuestas de ins- 
trumentos para facilitar medidas de eficiencia energética en el sector industrial de México. Fundación Bariloche. Recuperado de https://www.gob.mx/cms/uploads/attachment/file/400188/HojaRuta_EE_DIGITAL_.pdf

Cámara de Diputados del H. Congreso de la Unión (2008). Ley para el aprovechamiento sustentable de la energía. Nueva ley. DOF, 28 de noviembre de 2008. Recuperado de https://www.senado.gob.mx/comisiones/energia/docs/marco_LASE. pdf

CEPAL [Comisión Económica para América Latina y el Caribe] (1999). Las barreras medioambientales a las exportaciones latinoamericanas de camarón. Unidad de comercio internacional. Recuperado de https://www.cepal.org/es/publicaciones/4326-barreras-medioambientales-exportaciones-latinoamericanas-camarones

Conuee [Comisión Nacional para el Uso Eficiente de la Energía] (2018). Micro, pequeña y mediana empresa 2018. Recuperado de https://www.gob.mx/conuee/ acciones-y-programas/micro-pequena-y-mediana-empresa-2018 [consulta: $10 \mathrm{de}$ febrero de 2020].

Conuee [Comisión Nacional para el Uso Eficiente de la Energía] (2019). Hoy día, la eficiencia energética en México contribuye más a la mitigación del cambio climático que las energías renovables. Recuperado de https://www.gob.mx/conuee/articulos/hoy-dia-la-eficiencia-energetica-en-mexico-contribuye-mas-a-la-mitigacion-del-cambio-climatico-que-las-energias-renovables

Fawkes, S., Oung, K., Thorpe, D. (2016) Best practices and case studies for industrial energy efficiency improvement - An introduction for policy makers. Copenhagen: UNEP DTU Partnership. Recuperado de https://orbit.dtu.dk/files/127152472/Best_Practises_for_Industrial_EE_web_1_.pdf

Fischedick M., Roy, J., Abdel-Aziz, A., Acquaye, A., Allwood, J. M., Ceron, J.-P., Geng, Y., Kheshgi, H., Lanza, A., Perczyk, D., Price, L., Santalla, E., Sheinbaum, C. y Tanaka, K. (2014) Industry. En O. Edenhofer, R. Pichs-Madruga, Y. Sokona, E. Farahani, S. Kadner, K. Seyboth, A. Adler, I. Baum, S. Brunner, P. Eickemeier, B. Kriemann, J. Savolainen, S. Schlömer, C. von Stechow, T. Zwickel y J. C. Minx (eds.), Climate change 2014: Mitigation of climate change. Contribution of working group III to the fifth assessment report of the intergovernmental panel on climate change. Cambridge, United Kingdom: Cambridge University Press.

Golubchikov, O. y Deda, P. (2012). Governance, technology, and equity: An integrated policy framework for energy efficient housing. Energy Policy, 41, 733-741. DOI: https://doi.org/10.1016/j.enpol.2011.11.039 
González, D. y Martínez, M. (2012). Changes in CO2 emission intensities in the Mexican industry. Energy Policy, 51, 149-163. DoI: http://dx.doi.org/10.1016/j. enpol.2012.08.058

Herrera, B., Amell, A., Chejne, F., Cacua, K., Manrique, R., Henao, W. y Vallejo, G. (2017). Use of thermal energy and analysis of barriers to the implementation of thermal efficiency measures in cement production: Exploratory study in Colombia. Energy, 140, 1047-1058. DoI: https://doi.org/10.1016/j.energy.2017.09.041

IEA [International Energy Agency] (2012). Energy management programmes for industry. Paris, France. Recuperado de https://webstore.iea.org/policy-pathway-energy-management-programmes-for-industry

IEA [International Energy Agency] (2020a). Diagrama de Sankey de la Agencia Internacional de Energía. Recuperado de https://www.iea.org/sankey/

IEA [Inernational Energy Agency] (2020b). Data and statistics. Recuperado de https:// www.iea.org/data-and-statistics?country=WORLD\&fuel=CO2\%20emissions\&indicator $=\mathrm{CO} 2 \% 20$ emissions $\% 20$ by $\% 20$ sector

IEA [Inernational Energy Agency] (2021). Tracking Industry 2020. Recuperado de https://www.iea.org/reports/tracking-industry-2020 [consulta: 24 de agosto de 2021].

INEGI [Instituto Nacional de Estadística y Geografía] (2016). Estructura económica de Nuevo León en síntesis. Recuperado de http://internet.contenidos.inegi.org. $\mathrm{mx} /$ contenidos/Productos/prod_serv/contenidos/espanol/bvinegi/productos/ nueva_estruc/702825090029.pdf

INEGI [Instituto Nacional de Estadística y Geografía] (2009). Micro, pequeña, mediana y gran empresa. Recuperado de https://www.inegi.org.mx/contenidos/programas/ ce/2009/doc/minimonografias/m_pymes.pdf

IENGI. Directorio Estadístico Nacional de Unidades Económicas (DENUE). Recuperado de https://www.inegi.org.mx/app/mapa/denue/ (Consultado el 19 de febrero del 2020)

Levy, P. y Lemeshow, S. (2008). Sampling of populations: Methods and applications. Wiley: New Jersey.

Makridou, G., Andriosopoulos, K., Doumpos, M., y Zopounidis, C. (2016). Measuring the efficiency of energy-intensive industries across European countries. Energy Policy, 88, 573-583. DoI: http://dx.doi.org/10.1016/j.enpol.2015.06.042

Munguia, N., Vargas-Betancourt, N., Esquer, J., Giannetti, B. F., Liu, G., y Velazquez, L. E. (2018). Driving competitive advantage through energy efficiency in Mexican maquiladoras. Journal of Cleaner Production, 172, 3379-3386. Dor: https://doi. org/10.1016/j.jclepro.2017.11.253 
Nordelo, C. A. B., Pérez, M. M., Felipe, C. P. V., Delgado, N. O. y Soldatti, M. E. (2014). Barreras y factores claves para promover la eficiencia energética en la industria. Universidad y Sociedad, 6(2). Recuperado de https://rus.ucf.edu.cu/ index.php/rus/article/download/158/155

Nouri, J., Lotfi, F. H., Borgheipour, H., Atabi, F., Sadeghzadeh, S. M. y Moghaddas, $Z$. (2013). An analysis of the implementation of energy efficiency measures in the vegetable oil industry of Iran: a data envelopment analysis approach. Journal of Cleaner Production, 52, 84-93. DoI: http://dx.doi.org/10.1016/j.jclepro.2013.02.021

Ozawa, L., Sheinbaum, C., Martin, N., Worrell, E. y Price, L. (2002). Energy use and CO2 emissions in Mexico's iron and steel industry. Energy, 27(3), 225-239.

OECD, IEA y AFD [Organización para la Cooperación y el Desarrollo Económicos, International Energy Agency, Agence Française de Développement] (2008). Promoting energy efficiency investment Case studies in the residential sector. Autor.

Qi, X., Guo, P., Guo, Y., Liu, X. y Zhou, X. (2020). Understanding energy efficiency and its drivers: An empirical analysis of China's 14 coal intensive industries. Energy, 190, 116-354. DoI: https://doi.org/10.1016/j.energy.2019.116354

Ramírez, D. M. y Luyando, J. R. (2014). Análisis del consumo de energía eléctrica residencial en el Área Metropolitana de Monterrey, NL, México. Estudios Económicos, 31(62), 27-47.

Rohdin, P., Thollander, P. y Solding, P. (2007). Barriers to and drivers for energy efficiency in the Swedish foundry industry. Energy Policy, 35(1), 672-677. Dor: http:// doi:10.1016/j.enpol.2006.01.010

Ruchansky, B., Januzzi, G., Buen, O. D. y Romero, A. (2011). Eficacia institucional de los programas nacionales de eficiencia energética: los casos de Brasil, Chile, México y el Uruguay. CEPAL: Santiago de Chile.

Secretaría de Economía y Trabajo (2019). Data Nuevo León: Indicadores oportunos. Recuperado de http://datos.nl.gob.mx/ [consulta: 21 de febrero de 2020].

SENER [Secretaría de Energía] y Banco Mundial (2019). Evaluación rápida del uso de la energía, Monterrey, Nuevo León, México. Recuperado de https://www.gob.mx/cms/ uploads/attachment/file/171266/21__Monterrey.pdf

Sener y Conuee [Secretaría de Energía y Comisión Nacional para el Uso Eficiente de la Energía] (2017). Normas oficiales mexicanas de eficiencia energética. Balance al 2017. Recuperado de https://www.gob.mx/cms/uploads/attachment/file/445141/NOMENER Balance_2017_V_octubre_2018.pdf

SEner y Conuee [Secretaría de Energía y Comisión Nacional para el Uso Eficiente de la Energía] (2019a). Segundo reporte de evaluación sobre los Acuerdos Voluntarios 
2019. Recuperado de https://www.gob.mx/cms/uploads/attachment/file/481355/ Segundo_Reporte_AVEE_2019_29Julio2019_minima_1_.pdf

SENER y ConUeE [Secretaría de Energía y Comisión Nacional para el Uso Eficiente de la Energía] (2019b). Programa Nacional para Sistemas de Gestión de la Energía 2013-2018. Ciudad de México. Recuperado de https://www.gob.mx/cms/uploads/ attachment/file/457626/Informe_PRONASGEn_Final_30042019.pdf

SIEMENS (2010). Estudios de servicios energéticos en el sector terciario. Recuperado de https://www.fenercom.com/wp-content/uploads/2019/10/2010-02-17-Estudios-de-Servicios-Energeticos-en-el-Sector-Terciario-fenercom.pdf [consulta. 2 de febrero de 2020].

Sheinbaum, C., Ozawa, L. y Castillo, D. (2010). Using logarithmic mean Divisia index to analyze changes in energy use and carbon dioxide emissions in Mexico's iron and steel industry. Energy Economics, 32(6), 1337-1344. DoI: http://doi:10.1016/j. eneco.2010.02.011

Sheinbaum-Pardo, C., Mora-Pérez, S. y Robles-Morales, G. (2012). Decomposition of energy consumption and $\mathrm{CO} 2$ emissions in Mexican manufacturing industries: Trends between 1990 and 2008. Energy for Sustainable Development, 16(1), 57-67. DoI: https://doi:10.1016/j.esd.2011.08.003

Sola, A. V. y Mota, C. M. (2020). Influencing factors on energy management in industries. Journal of Cleaner Production, 248, 119263. DoI: https://doi.org/10.1016/j. jclepro.2019.119263

Subsecretaria de Energías Renovables y Eficiencia Energética (2019). Reporte energético preliminar. Secretaria de Energía, Argentina. Recuperado de https://bneu.eficienciaenergetica.net.ar/Reporte_Energetico_Preliminar_190927.pdf

Tanaka, K. (2011). Review of policies and measures for energy eficiency in industry sector. Energy Policy, 39, 6532-6550. DoI: https://doi.org/10.1016/j.enpol.2011.07.058

Thollander, P., Backlund, S., Trianni, A. y Cagno, E. (2013). Beyond barriers -A case study on driving forces for improved energy efficiency in the foundry industries in Finland, France, Germany, Italy, Poland, Spain, and Sweden. Applied Energy, 111, 636-643. DoI: http://dx.doi.org/10.1016/j.apenergy.2013.05.036

Timilsina, G. R., Hochman, G. y Fedets, I. (2016). Understanding energy efficiency barriers in Ukraine: Insights from a survey of commercial and industrial firms. Energy, 106, 203-211. Dor: http://dx.doi.org/10.1016/j.energy.2016.03.009

Wang, J., Yang, F., Zhang, X. y Zhou, Q. (2018). Barriers and drivers for enterprise energy efficiency: An exploratory study for industrial transfer in the Beijing-Tianjin-Hebei region. Journal of Cleaner Production, 200, 866-879. Dor: https:// doi.org/10.1016/j.jclepro.2018.07.327 
Xue, X., Wu, H., Zhang, X., Dai, J. y Su, C. (2015). Measuring energy consumption efficiency of the construction industry: the case of China. Journal of Cleaner Production, 107, 509-515. DoI: http://dx.doi.org/10.1016/j.jclepro.2014.04.082

Zhou, Z., Xu, G., Wang, C. y Wu, J. (2019). Modeling undesirable output with a DEA approach based on an exponential transformation: An application to measure the energy efficiency of Chinese industry. Journal of Cleaner Production, 236, 117717. DoI: https://doi.org/10.1016/j.jclepro.2019.117717

Zuberi, M. J. S., Santoro, M., Eberle, A., Bhadbhade, N., Sulzer, S., Wellig, B. y Patel, M. K. (2020). A detailed review on current status of energy efficiency improvement in the Swiss industry sector. Energy Policy, 137, 111162. Dor: https://doi. org/10.1016/j.enpol.2019.111162 